\title{
Mathematical Modeling of Non-Selective Channels: Estimating Ion Current Fractions and Their Impact on Pathological Simulations
}

\author{
Eike M Wülfers, Peter Kohl, Gunnar Seemann \\ Institute for Experimental Cardiovascular Medicine, \\ University Heart Center Freiburg - Bad Krozingen, Medical Center, University of Freiburg, \\ and Faculty of Medicine, University of Freiburg, Freiburg, Germany
}

\begin{abstract}
Currents through non-selective ion channels are often mathematically modeled as an Ohmic current. In such models, quantifying the contributions of different contributing ion species is not easily possible.

We present a method to adapt Ohmic descriptions using the Goldman-Hodgkin-Katz equation in order to describe every ion species' contribution to the total channel current. We use our method to adapt a model of Channelrhodopsin2, a light-gated cation non-selective channel, and test our adaption in single cell and 1-dimensional tissue strand simulations of ventricular tachycardia.

Resulting contribution ratios of sodium, potassium and proton currents match expectations and correlate well with previously published data. Simulations of optical defibrillation for ventricular tachycardia show that our model predicts a detrimental outcome in ischaemia-like pathological settings that are missed by the original (Ohmic) model.
\end{abstract}

\section{Introduction}

Non-selective channels (NSC) are a category of ion channels that allow different types of ions to pass through them. NSC include physiologically relevant channels such as stretch-activated channels and gap-junctions, but also channels that can be expressed genetically and are useful in experimental settings. Recent studies have, for instance, shown optical defibrillation in mice using Channelrhodopsin-2 (ChR2), a light-gated cation NSC [1].

Many mathematical models of NSC describe the channel current density based on Ohm's law in the form

$$
I_{N S C, O h m}=g_{N S C} \cdot O_{N S C} \cdot\left(V_{m}-E_{N S C}\right) .
$$

The current depends on a channel conductivity $g_{N S C}$, the transmembrane voltage $V_{m}$, and the channel's equilibrium potential $E_{N S C}$. It is optionally modulated by a gating mechanism $O_{N S C}$, for instance modeled based on patch- clamp data and often mathematically described by ordinary differential equations.

Formulations based on Ohm's law only describe the total electric current, which is the sum of all ion currents passing through the channel. The contribution of any ion species passing the channel can not easily be deduced from this equation, since it depends on the selective permeability, concentration gradient, and membrane voltage.

The Ohmic representation is often sufficient to describe effects of NSC on cellular electrophysiology by the mechanisms of short-term de-/repolarization, small leak-like currents, etc. [2,3]. However, NSC effects on cellular ion concentrations may be functionally relevant, both during long-term NSC activation, or in pathological settings of reduced electrophysiological stability.

Most mathematical models of cellular electrophysiology that are able to simulate pathological conditions include sodium, potassium, and calcium concentrations as state variables. Realistic modeling of NSC effects on pathologies therefore requires inclusion of the influence that these channels have on cellular ion concentrations. Here, we present a method to adapt channel models based on Ohm's law in a way that allows one to describe currents of every ion species passing through the channel. We show that neglect to consider these ionic effects may give rise to misleading interpretations in models of pathological conditions.

\section{Methods}

\subsection{Mathematical NSC Model}

NSC in the open state can be approximated as pores in the membrane. The ion current of an ion species $X$ through any such pore is described by the Goldman-Hodgkin-Katz (GHK) equation as

$$
I_{X}=\frac{V_{m} F^{2}}{R T} P_{X} z_{X}^{2} \frac{[X]_{i}-[X]_{e} \exp \left(-z_{X} V_{m} F / R T\right)}{1-\exp \left(-z_{X} V_{m} F / R T\right)},
$$


with $[X]_{i}$ and $[X]_{e}$ intra- and extracellular ion concentrations, and $P_{X}$ and $z_{X}$ permeability and valence of $X$, respectively [4]. It follows that $I_{N S C, G H K}=\sum_{X} I_{X}$ for all ions $X$ that contribute to the ion current through the channel. Assuming there exists an Ohmic model $I_{N S C, O h m}$ for a NSC as described above, we can then choose unknown parameters in $I_{N S C, G H K}$ such that $I_{N S C, G H K} \approx$ $I_{N S C, O h m}$. The so parameterized model would follow a similar trajectory as the original Ohmic model, but would also allow quantification of the contribution of all ions $X$.

We apply this method to adapt a model of ChR2 by Williams et al. [5], where (simplified)

$$
I_{C h R 2}=g_{C h R 2} \cdot O \cdot\left(V_{m}-E_{C h R 2}\right) .
$$

We consider the cations $\mathrm{H}^{+}, \mathrm{Na}^{+}, \mathrm{K}^{+}$, and $\mathrm{Ca}^{2+}$. Measured data is available for $P_{K}$ and $P_{C a}$ relative to $P_{N a}$, and the ratio $P_{H} / P_{N a}$ has been calculated based on the measured equilibrium potential [6]. We are then left with one unknown, $P_{N a}$. To approximate the Ohmic model, we solve

$$
g_{C h R 2}=\lim _{V_{m} \longrightarrow 0} \frac{\mathrm{d} I_{C h R 2, G H K}}{\mathrm{~d} V_{m}}
$$

in order to match the Ohmic current-voltage relationship in slope at $0 \mathrm{mV}$ (approximately the equilibrium potential). This leads to

$$
\begin{aligned}
P_{N a}=2 g_{C h R 2} \frac{R T}{F^{2}}\left(\left[N a^{+}\right]_{i}+\left[N a^{+}\right]_{e}+\right. \\
\frac{P_{H}}{P_{N a}}\left(\left[H^{+}\right]_{i}+\left[H^{+}\right]_{e}\right)+\frac{P_{K}}{P_{N a}}\left(\left[K^{+}\right]_{i}+\left[K^{+}\right]_{e}\right)+ \\
\left.4 \frac{P_{C a}}{P_{N a}}\left(\left[\mathrm{Ca}^{2+}\right]_{i}+\left[C a^{2+}\right]_{e}\right)\right)^{-1} .
\end{aligned}
$$

\subsection{Ventricular Tachycardia Model}

Pathological scenarios were explored in a 1-dimensional (1D) strand of $10 \mathrm{~cm}$ length at $0.1 \mathrm{~mm}$ resolution. Simulations were conducted using our software "acCELLerate" with a fixed integration time step of $0.01 \mathrm{~ms}$.

We used the epicardial variant of the ten Tusscher \& Panfilov human ventricular myocyte model (TP06) with some modifications to represent pathological substrate [7]. Namely, based on [8], $g_{K r}$ was varied between $0 \%$ and $100 \%$ of its original value to represent varying degrees of long-QT (LQT) syndrome. Likewise, $g_{\mathrm{CaL}}$ was varied between $100 \%$ and $1000 \%$ while $\tau_{f}$ was set to either $50 \%$ or $100 \%$ to increase the probability of early afterdepolarizations (EAD), which are suspected to cause VT in LQT patients. A reduction of $\tau_{f}$ to $50 \%$ must, however, be compensated by doubling $g_{C a L}$, meaning that $1000 \% g_{C a L}$ at $50 \% \tau_{f}$ are equivalent to a five-fold increase of $I_{C a L}$. The cell model was initialized from steady state values resulting from single-cell pacing simulations. Parameter sets
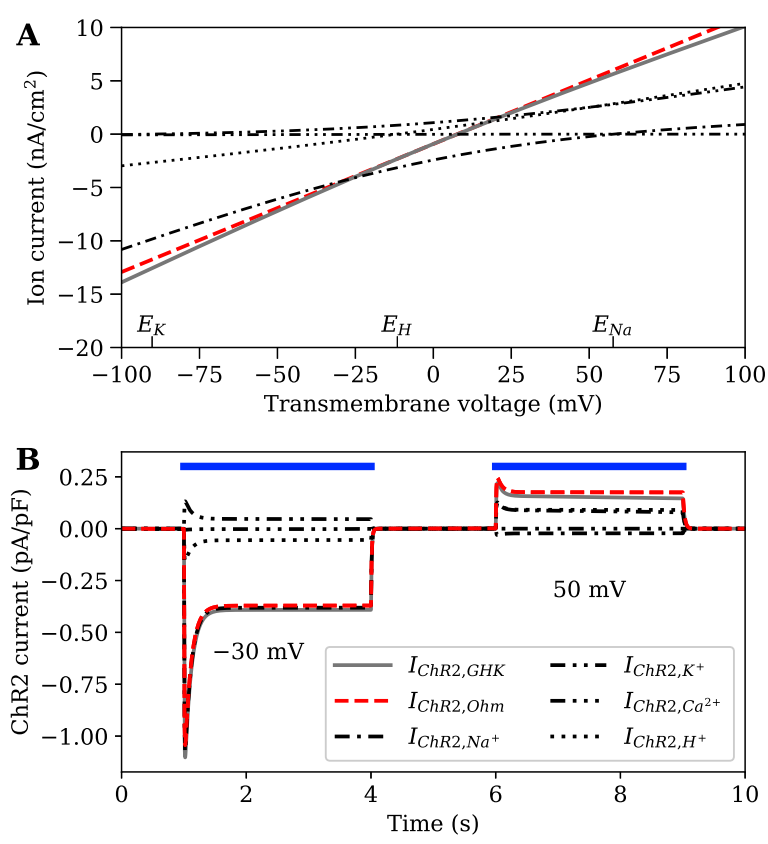

Figure 1. A Current-voltage relationship for the two ChR2 models at physiological ion concentrations. $g_{C h R 2}=$ $0.12 \mathrm{mS} / \mathrm{cm}^{2}$, no gating. B Patch-clamp simulations comparing Ohmic and adapted ChR2 model at $-30 \mathrm{mV}$ and $50 \mathrm{mV}$. Blue bar at top indicates illumination $\left(0.5 \mathrm{~mW} / \mathrm{mm}^{2}, 470 \mathrm{~nm}, g_{C h R 2}=0.12 \mathrm{mS} / \mathrm{cm}^{2}\right.$.

that did not result in a periodic steady state after pacing for $1000 \mathrm{~s}$ were discarded.

Acute VT was simulated by rapidly pacing the strand at $2 \mathrm{~Hz}$ or $3 \mathrm{~Hz}$ for $10 \mathrm{~s}$. Optical defibrillation was simulated by illuminating the whole strand for $10 \mathrm{~s}$ at $10 \mathrm{~mW} / \mathrm{mm}^{2}$. After that, the strand was stimulated from one side at $1 \mathrm{~Hz}$ to simulate excitation in sinus rhythm. We then investigated for which sets of parameters sinus rhythm could be maintained for $30 \mathrm{~s}$, and whether the choice of ChR2 model variant (Ohmic or GHK) would alter the outcome. In particular, we were interested in cases where one model variant would predict normal sinus propagation while the other variant suggested recurrence of arrhythmia.

\section{Results}

\subsection{Adaption of ChR2 Model}

We calculated $P_{N a}$ using (1) with the measured permeabilities from [6] and default ion concentrations from the TP06 model. Fig. 1A shows the resulting ion currents compared to the Ohmic relationship from [5] at physiological ion concentrations. Both, the so parameterized channel model and the original formulation by Williams et al. were 

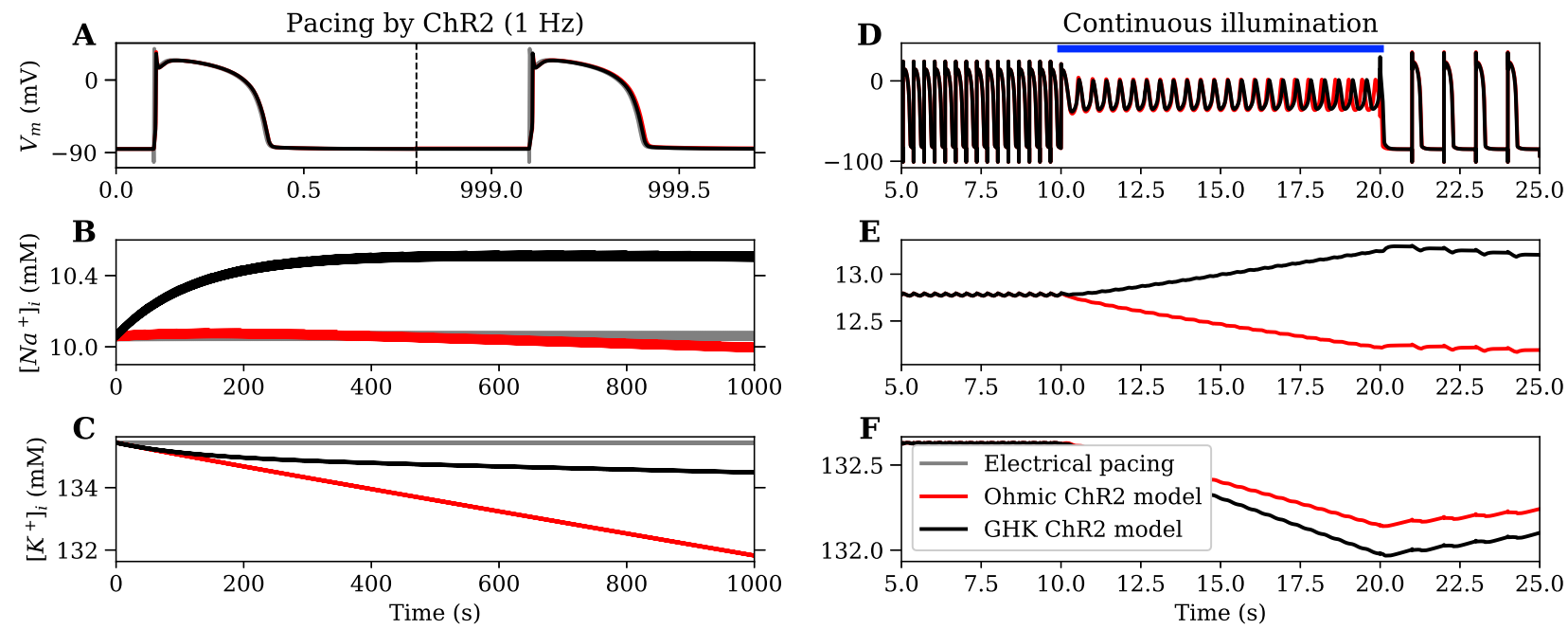

Figure 2. A-C Results from pacing simulations (1 Hz for $1000 \mathrm{~s})$. A Overlay of first and last action potentials from all stimulation modi. B, C Intracellular sodium and potassium concentration, respectively, during $1000 \mathrm{~s}$ of pacing. DF Results from continuous illumination (“defibrillation”) after rapid pacing (3 Hz). D Transmembrane voltages, blue bar at top indicates active illumination. E, F Intracellular sodium and potassium concentrations, respectively.

integrated into the TP06 model.

Fig. 1B shows results from two exemplary patch-clamp simulations at different holding potentials. Unsurprisingly, sodium ions constituted the majority of the current at a negative holding potential, while potassium ions and protons did so at a positive holding potential. The contribution ratios of the several ion species at different concentrations and voltages correlated well with another model of ChR2 that describes these ion currents separately [9].

A possible application of light-gated channels such as ChR2 is contactless pacing of the heart. We investigated optical pacing in single-cell simulations using the TP06 model. The model was first paced electrically (biphasic, $1 \mathrm{~Hz}, 4 \mathrm{nA}, 3 \mathrm{~ms}$ pulse duration) until a steady state was reached. Then, the model was paced for $1000 \mathrm{~s}$ at $1 \mathrm{~Hz}$ using either ChR2 formulation $\left(1 \mathrm{~Hz}, 5.5 \mathrm{~mW} / \mathrm{mm}^{2}, 470\right.$ $\mathrm{nm}$ wavelength, $5 \mathrm{~ms}$ pulse duration).

Fig. 2A shows that after $1000 \mathrm{~s}$ there were virtually no differences in AP morphology between pacing optically, using either ChR2 model, and continued electrical pacing. However, $\left[\mathrm{Na}^{+}\right]_{i}$ is predicted to be mostly stable using the original ChR2 model (similar to electric pacing, cf. fig. 2B). Our GHK-based model on the other hand predicts an increase in intracellular sodium (as expected at physiological ion concentrations). The charge imbalance caused by the purely electrical ChR2 current of the Williams et al. model causes a monotonic reduction of intracellular potassium in the cell model (fig. 2C). Using our model, $\left[\mathrm{K}^{+}\right]_{i}$ is reduced only slightly, which fits expectations: The equilibrium potential of potassium is around $-90 \mathrm{mV}$ and, when used for pacing, ChR2 will mostly be open while the cell's transmembrane voltage is close to that.

Since several studies suggested using ChR2 for optical "defibrillation" (i.e., temporary silencing of electrically active tissue), we conducted a simulation to investigate the effects of $10 \mathrm{~s}$ illumination $\left(5.5 \mathrm{~mW} / \mathrm{mm}^{2}, 470 \mathrm{~nm}\right)$ after rapid pacing $(3 \mathrm{~Hz})$ on cellular electrophysiology (fig. 2DF). Fig. 2E shows how $\left[\mathrm{Na}^{+}\right]_{i}$ develops in different directions during defibrillation: The model by Williams et al. leads to a reduction of intracellular sodium in the TP06 model, whereas our model predicts an increase. During continuous illumination, the transmembrane voltage oscillates without pacing. After defibrillation, electrical pacing resumed at $1 \mathrm{~Hz}$ and the models showed no significant difference in their action potentials despite the different $\left[\mathrm{Na}^{+}\right]_{i}$.

\subsection{VT and Defibrillation Model}

In the single cell simulations, the two ChR2 model variants predicted opposite effects of longer-lasting periods of illumination on $\left[\mathrm{Na}^{+}\right]_{i}$. Acknowledging that this effect could be critical under pathological conditions, we tested both variants in the 1D LQT strand model described above. Similar to the single cell experiments, $\left[\mathrm{Na}^{+}\right]_{i}$ dropped during $10 \mathrm{~s}$ illumination using the original formulation, while it rose when using our GHK-based model. In certain conditions, the original formulation predicted normal excitation conduction after optical defibrillation, while our model predicted spontaneous EADs that lead to blocks or 

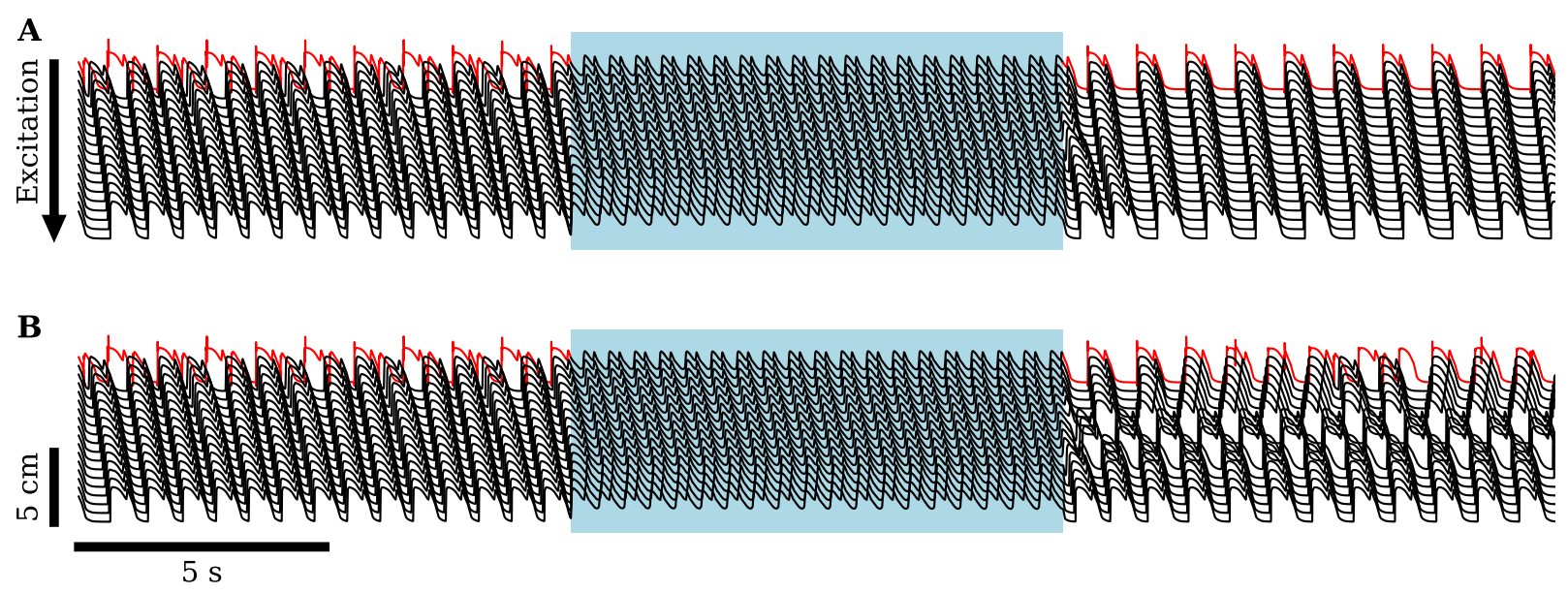

Figure 3. Transmembrane voltage plots showing excitation conduction through a 1D strand. $10 \mathrm{~s}$ of VT (by electrical pacing at $3 \mathrm{~Hz}$ from one side (red)) are followed by $10 \mathrm{~s}$ of continuous illumination of the whole strand (blue background), activating ChR2. After that, pacing resumes at $1 \mathrm{~Hz}$. Simulation shown in $\mathbf{A}$ used the Ohmic ChR2 formulation from Willams et al., B used our GHK variant. Cell model modifications: $90 \% g_{K r}, 50 \% \tau_{f}, 900 \% g_{C a L}$.

reflections disturbing the normal rhythm, aggravating VT (cf. fig. 3A and B).

\section{Conclusions}

We present a method to adapt mathematical models of non-selective ion channels in a way that preserves their current-voltage relationship while allowing for quantification of partial currents associated with different ion species. We show that changes in intracellular ion concentrations are affected significantly by the choice of model, and that consideration of ion concentration changes may give rise to to different outcomes in VT defibrillation simulations. Our simulations also indicate that optical defibrillation would be improved by using a truly repolarizing (e.g., $K^{+}$selective) channel rather than a depolarizationbased approach to silencing electrical activity.

\section{Acknowledgements}

The authors gratefully acknowledge funding from the ERC (Advanced Grant CardioNECT) and DFG (Proj. No. 183027722).

\section{References}

[1] Bruegmann T, Beiert T, Vogt CC, Schrickel JW, Sasse P. Optogenetic termination of atrial fibrillation in mice. Cardiovasc Res 2017;114(5):713.

[2] Williams JC, Entcheva E. Optogenetic versus electrical stim- ulation of human cardiomyocytes: Modeling insights. Biophys J 2015;108(8):1934.

[3] Kohl P, Hunter P, Noble D. Stretch-induced changes in heart rate and rhythm: clinical observations, experiments and mathematical models. PBMB 1999;71(1):91.

[4] Hille B. Ionic CHannels of Excitable Membranes. 2nd edition. Sunderland, MA: Sinauer Associates Inc., 1992.

[5] Williams JC, Xu J, Lu Z, Klimas A, Chen X, Ambrosi CM, Cohen IS, Entcheva E. Computational Optogenetics: Empirically-Derived Voltage- and Light-Sensitive Channelrhodopsin-2 Model. PLoS Comp Biol 2013; 9(9):e1003220.

[6] Lin JY, Lin MZ, Steinbach P, Tsien RY. Characterization of engineered channelrhodopsin variants with improved properties and kinetics. Biophys J 2009;96(5):1803.

[7] ten Tusscher KHWJ, Panfilov AV. Alternans and spiral breakup in a human ventricular tissue model. Am J Physiol Heart and Circ Physiol 2006;291(3):H1088.

[8] Vandersickel N, Kazbanov IV, Nuitermans A, Weise LD, Pandit R, Panfilov AV. A study of early afterdepolarizations in a model for human ventricular tissue. PLoS ONE 2014; $9(1)$.

[9] Schneider F, Gradmann D, Hegemann P. Ion selectivity and competition in channelrhodopsins. Biophys J 2013; 105(1):91.

Address for correspondence:

Eike M. Wülfers

Institute for Experimental Cardiovascular Medicine

Elsässer Str. 2Q, 79110 Freiburg, Germany.

eike.wuelfers@universitaets-herzzentrum.de 